\title{
Comparação da qualidade de vida entre idosos que participam e idosos que não participam de grupos de convivência na cidade de Itabira-MG
}

\author{
Comparison of quality of life among elderly that participate and do not
}

participate of senior conviviality groups in the city of Itabira-MG, Brazil

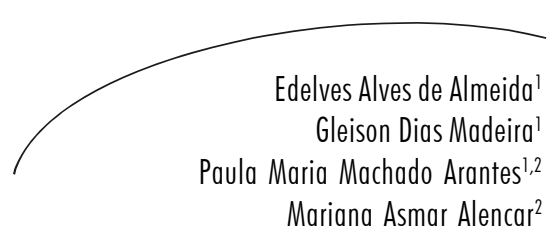

Resumo

Com o aumento no número de idosos no Brasil e no mundo, torna-se preocupante a qualidade de vida dos idosos acima de 60 anos. Diante disto, uma forma que se existe atualmente para que o idoso tente melhorar sua qualidade de vida é sua participação em grupos de convivência. Objetivos: Avaliar a qualidade de vida e a presença de transtorno depressivo entre idosos participantes e não participantes em grupos de convivência. Métodos: A amostra foi composta por 30 idosos que participam de grupos de convivência $(66,73+5,82$ anos) e 30 idosos que não participam de grupo de convivência $(66,70+5,70$ anos). Para avaliar a qualidade de vida, foi utilizado o questionário genérico Medical Outcomes Study 36 - Item Short-Form Health Survey e para rastrear a presença de transtorno depressivo utilizou-se a Escala de Depressão Geriátrica. Para verificar a normalidade entre os grupos, foi utilizado o teste de Shapiro-Wilk. O teste $t$-Student e o teste Mann-Whitney $U$ para amostras independentes foram utilizados para realizar a comparação entre os grupos. O nível de significância foi mantido em 0,05 para todas as análises. Resultados: Foi encontrada diferença estatisticamente significativa nos domínios capacidade funcional, estado geral de saúde, vitalidade e aspectos sociais. Conclusões: Os resultados sugerem que os idosos que participam de grupos de convivência apresentam melhor qualidade de vida e menor ocorrência de depressão quando comparados a idosos que não participam de grupos de terceira idade.

\section{Abstract}

With the increase in the number of elderly people in Brazil and abroad, the quality of life of elderly over 60 years is worrisome. So, a way that can help the elderly try to improve their quality of life is their participation in conviviality groups. Objective: The purpose of this study is to assess the quality of life and the presence of depressive disorder among elderly participants and non participants in conviviality groups. Methodology: The sample consisted of 30 elderly involved conviviality groups $(66,73 \pm 5,82$ years $)$ and 30 elderly people who do not participate in a group of

\footnotetext{
1 Fundação Comunitária de Ensino Superior de Itabira. Curso de Fisioterapia. Itabira, MG, Brasil.

2 Universidade Federal de Minas Gerais. Programa de Pós-graduação em Ciências da Reabilitação. Belo Horizonte, MG, Brasil

Palavras-chave: Qualidade de Vida. Idosos. Grupos de Convivência. SF-36. GDS. 
coexistence $(66,70 \pm 5,70$ years). To assess the quality of life questionnaire it was used the generic SF-36, and to trace the presence of depressive disorder, it was used the GDS. To check the normality between the groups, it was used the Shapiro-Wilk test. The test for independent samples t-Student and the Mann-Whitney U test were used to perform the comparison between groups. The level of significance was maintained at 0.05 for all tests. Results: It was found a statistically significant difference in the functional capacity, general health, vitality and social aspects. Conclusions: The results suggest that older people that participate in conviviality groups have a better quality of life and lower incidence of depression when compared to older people who do not participate in a senior conviviality group.
Key words: Quality of Life. The Elderly. Groups of Living. SF-36. GDS.

\section{INTRODUÇÃO}

Com o aumento da expectativa de vida da população, tornam-se predominantes as doenças crônico-degenerativas e suas complicações, como a perda da autonomia e independência funcional, sendo um desafio para a sociedade e o sistema de saúde ${ }^{1-4}$. Para Gordilho et al. ${ }^{5}$, a maioria das doenças crônicas que acometem o idoso tem, na própria idade, seu principal fator de risco. Referem-se a doenças crônicas - como câncer, diabetes, hipertensão arterial, doença coronariana e cerebrovascular, Parkinson e outros problemas do sistema nervoso, hepatite, artrites crônicas e doenças respiratórias - ou a consequências crônicas de doenças ou agravos agudos, como problemas neurológicos pós-traumáticos, transplantes, e medicamentos de uso prolongado ${ }^{5-}$ 7. Uma vez presente, a doença crônica está associada à piora da qualidade de vida ${ }^{8,9}$.

Além das doenças crônicas, a inatividade, o aumento da dependência em executar as AVDs e a falta de perspectivas na aposentadoria podem desencadear um alto nível de ansiedade e depressão ${ }^{10}$, comprometendo a qualidade de vida dos idosos ${ }^{11}$, Assim, é de extrema importância que sejam desenvolvidas estratégias que garantam aos idosos não apenas maior longevidade, mas felicidade, satisfação pessoal e qualidade de vida ${ }^{12}$.

A qualidade de vida foi definida pelo Grupo de Qualidade de Vida da Organização Mundial de Saúde ${ }^{13}$ como "percepção do indivíduo de sua posição na vida, no contexto da cultura e sistema de valores nos quais ele vive e em relação aos seus objetivos, expectativas, padrões e preocupações" ${ }^{\prime 14}$. É um conceito subjetivo dependente do nível sociocultural, da faixa etária e das aspirações pessoais do indivíduo. Está relacionado à autoestima e ao bem-estar pessoal e abrange uma série de aspectos como a capacidade funcional, o nível socioeconômico, o estado emocional, a interação social, o autocuidado, o suporte familiar, o próprio estado de saúde, os valores éticos e religiosos, o estilo de vida, a satisfação com as atividades diárias e o meio em que o indivíduo está inserido ${ }^{15-18}$.

Frutuoso ${ }^{19}$ relata que muitos estudos enfocam um aumento na qualidade de vida e da longevidade em idosos que mantêm uma vida social intensa, sugerindo que relacionamentos sociais são fatores importantes para o bem-estar físico e mental na velhice. A ideia de formar grupos de idosos está se propagando em todas as regiões do país ${ }^{20}$. Interagir socialmente, sobretudo com amigos da mesma geração $0^{21}$, possibilita ao idoso construir novos laços de relação ${ }^{22} \mathrm{e}$ favorece o bem-estar físico, psicológico e social dos idosos ${ }^{21}$. As deficiências em habilidades sociais parecem constituir um fator de vulnerabilidade para a baixa qualidade de vida e para a depressão em indivíduos da terceira idade ${ }^{23}$.

Segundo Capitanini ${ }^{24}$, embora a solidão se torne uma ocorrência mais possível na velhice, pode-se envelhecer sem solidão ou isolamento. Quanto mais atuantes e integrados em seu meio social, menos ônus trarão para a família e para os serviços de saúde ${ }^{25}$. As pessoas que têm maior contato social vivem mais e com melhor saúde quando comparadas às pessoas com menor contato social ${ }^{26}$. A pobreza de relações sociais tem sido considerada um fator de risco à saúde, tão danoso quanto o fumo, a pressão arterial elevada, 
a obesidade e a ausência de atividade física ${ }^{15}$. Parece que a capacidade de interagir socialmente é fundamental para o idoso, para conquistar e manter redes de apoio social e, consequentemente, garantir melhor qualidade de vida ${ }^{27}$.

Embora a literatura enfatize a importância da manutenção da qualidade de vida do idoso e das medidas disponíveis para contribuir para a melhora desta, são escassos os estudos que investigam a qualidade de vida em grupos de convivência. São escassos também estudos que avaliem a relação entre a presença de depressão e a participação dos idosos nesses grupos de convivência. Assim, o presente estudo teve como objetivo avaliar a qualidade de vida e a presença de depressão em idosos que participam e idosos que não participam de grupos de convivência na cidade de Itabira-MG.

\section{MÉTODO}

\section{Participantes}

A amostra do grupo 1 (G1) consistiu de 30 idosos (23 do sexo feminino e 7 do sexo masculino), que frequentavam quatro grupos de convivência do Projeto Vida Ativa na cidade de Itabira. Esses grupos de convivência estavam cadastrados na Secretaria Municipal de Esporte e Lazer da cidade de Itabira, Minas Gerais. A amostra do grupo 2 (G2) consistiu de 30 idosos (17 do sexo feminino e 13 do sexo masculino). Para determinação do número de participantes, foi realizado cálculo de tamanho da amostra considerando a $=0,05$ e poder estatístico de $80 \%$.

Os critérios de inclusão foram: idade igual ou superior a 60 anos, viver na comunidade e ser sedentário de acordo com os critérios do American College of Sports Medicine ${ }^{28}$. Para fazer parte do grupo G1, os idosos deveriam frequentar o grupo de convivência há mais de seis meses. Para ser incluído no grupo G2 o idoso não poderia fazer parte de nenhum grupo de convivência. Foram critérios para exclusão do estudo: presença de déficit cognitivo que comprometia a compreensão dos testes, doenças neurológicas e ser cadeirante.
Os participantes dos dois grupos foram pareados pela idade de acordo com grupos etários com intervalos de três anos.

\section{Instrumentação}

Uma Ficha de Avaliação Inicial foi utilizada para a obtenção de dados pessoais referentes à amostra estudada como idade, sexo, escolaridade, dados de saúde atuais, medicamento em uso, situação ocupacional, composição familiar, tempo de frequência e grau de satisfação com o Núcleo (G1).

O protocolo do estudo constou de uma entrevista com aplicação do SF-36 e Escala de Depressão Geriátrica.

O SF-36 (Medical Outcomes Study 36 - Item ShortForm Health Survey) é um instrumento genérico de avaliação de qualidade de vida, de fácil administração e compreensão. É formado por 36 itens, englobados em oito escalas ou componentes: capacidade funcional (desempenho das atividades diárias, como capacidade de cuidar de si, vestirse, tomar banho e subir escadas); aspectos físicos (impacto da saúde física no desempenho das atividades diárias e ou profissionais); dor (nível de dor e o impacto no desempenho das atividades diárias e ou profissionais); estado geral de saúde (percepção subjetiva do estado geral de saúde); vitalidade (percepção subjetiva do estado de saúde); aspectos sociais (reflexo da condição de saúde física nas atividades sociais); aspectos emocionais (reflexo das condições emocionais no desempenho das atividades diárias e ou profissionais) e saúde mental (escala de humor e bem-estar). Apresenta um escore final de $\mathrm{O}$ a 100, no qual zero corresponde a pior estado geral de saúde e 100 a melhor estado de saúde ${ }^{8,29}$.

Para a triagem de um possível transtorno depressivo, foi utilizado o instrumento Escala de Depressão Geriátrica de Yesavage, versão simplificada com 15 perguntas, recomendada pela Organização Mundial de Saúde - CID-1013,30. Nesta escala, as respostas são classificadas em sim ou não, sendo o escore total o somatório das respostas assinaladas nos 15 itens. Uma pontuação 
acima de cinco de pontos indica possível transtorno depressivo. Este ponto de corte produziu índices de sensibilidade de $85,4 \%$ e de especificidade de $73,9 \%$ para o diagnóstico de episódio depressivo maior de acordo com a CID$10^{31}$ e de $90,9 \%$ e $64,5 \%$, respectivamente, de acordo com os critérios da DSM-IV ${ }^{32}$. A versão utilizada foi adaptada por Yesavage et al. ${ }^{33}$, sendo considerada uma medida válida e confiável para uso em amostra brasileira ${ }^{34,35}$.

\section{Procedimento}

Cada participante recebeu o Termo de Consentimento Livre e Esclarecido, contendo informações sobre os objetivos da pesquisa e o compromisso com o sigilo a respeito da identidade dos participantes. Os voluntários que concordaram em participar do estudo o assinaram. Todos receberam cópia do formulário.

Os voluntários do G1 foram orientados a comparecerem a um local reservado no próprio ambiente do grupo de convivência, onde receberam uma explicação mais detalhada sobre os objetivos e procedimentos da pesquisa e o sigilo a respeito da sua identidade.

O recrutamento do G2 foi feito através de contato informal com pessoas que conheciam algum idoso nestas condições. Os indivíduos receberam a mesma explicação, mas esta foi realizada em seus próprios domićlios, com data e hora marcadas pelo voluntário.

As entrevistas foram realizadas de forma individual e com duração de aproximadamente 40 minutos. Inicialmente foram coletadas informações sobre os aspectos clínicos e demográficos. Em seguida, foram aplicados os instrumentos SF-36 e a escala GDS. A aplicação foi realizada por dois examinadores. Os examinadores receberam um treinamento prévio para a aplicação dos instrumentos e foi realizada análise de confiabilidade interexaminadores para todos os instrumentos aplicados. A confiabilidade no estudo-piloto variou de boa a excelente $(\mathrm{ICC}=0,82 \text { a } \mathrm{ICC}=0,92 ; \mathrm{p}<0,05)^{36}$.

Este estudo foi aprovado pelo Comitê de Ética em Pesquisa da Universidade Federal de Minas Gerais (COEP/UFMG), Parecer nº 0350/08.

\section{Análise estatística}

Para a análise estatística, foi utilizado o programa SPSS (versão 14.0 para Windows). Foi realizada análise descritiva de todos os dados coletados. Para verificar a normalidade da distribuição dos dados, foi utilizado o teste de Shapiro-Willk. O teste $t$-Student para amostras independentes foi utilizado para comparação do desfecho vitalidade entre os grupos, uma vez que este foi o único desfecho que apresentou distribuição normal. Para os demais desfechos, foi utilizado o teste Mann-Whitney Upara realizar as comparações entre os grupos. Para a comparação das variáveis categóricas, foram utilizados o teste Qui-Quadrado e o Teste Exato de Fisher. Em todas as análises, o nível de significância foi mantido em 0,05.

\section{RESULTADOS}

Os voluntários do grupo $\mathrm{G} 1$ tiveram média de idade $66,73+5,82$, e os do grupo G2, $66,70 \pm 5,70$, não havendo diferença significativa entre os grupos. Não houve diferença estatisticamente significativa entre os dois grupos, ao se comparar os demais aspectos demográficos e clínicos coletados. Estas características estão descritas na tabela 1 . 
Tabela 1 - Estatística descritiva dos dados demográficos e clínicos dos participantes com valores expressos em média, desvio-padrão e ocorrência. Itabira, MG, 2008.

\begin{tabular}{lrrr}
\hline \multicolumn{1}{c}{ Variável } & \multicolumn{2}{c}{ Média (DP) } & \\
& $\mathrm{G}$ G1 & \multicolumn{1}{c}{$\mathrm{G} 2$} & $\mathrm{p}$ \\
\hline Idade (anos) & $66,73(5,82)$ & $66,70(5,70)$ & 0,970 \\
Número de medicamentos & $2,27(1,53)$ & $3,27(3,41)$ & 0,242 \\
Alterações circulatórias & $11(37 \%)$ & $12(40 \%)$ & 0,791 \\
Alterações ortopédicas & $7(24 \%)$ & $11(36 \%)$ & 0,258 \\
Alterações musculares & $1(3 \%)$ & $2(6 \%)$ & 0,550 \\
Diabetes & $2(7 \%)$ & $3(10 \%)$ & 0,639 \\
Alterações visuais & $27(90 \%)$ & $25(84 \%)$ & 0,706 \\
Alterações pulmonares & $2(6 \%)$ & $2(6 \%)$ & 1 \\
Alterações auditivas & $4(13 \%)$ & $5(16 \%)$ & 1 \\
Vertigens, tonturas & $7(23 \%)$ & $7(23 \%)$ & 1 \\
Quedas & $5(16 \%)$ & $6(20 \%)$ & 1 \\
\hline
\end{tabular}

$\mathrm{n}=$ número de indivíduos

Os resultados descritivos por domínio doSF-36 e GDS podem ser observados na tabela 2. Pode-se observar que o grupo G1 apresentou médias superiores às do grupo $\mathrm{G} 2$ para todos dos domínios do SF-36, alcançando significância estatística nos domínios capacidade funcional $(p=0,000)$, estado geral de saúde $(p=0,004)$, vitalidade $(p=0,014)$, aspectos sociais $(\mathrm{p}=0,005)$ e saúde mental $(\mathrm{p}=0,035)$.

Tabela 2 - Comparação dos escores das dimensões do questionário do SF-36 e do GDS com valores expressos em média e desvio-padrão. Itabira, MG, 2008.

\begin{tabular}{lccc}
\hline \multicolumn{1}{c}{ Variável } & $\mathrm{G} 1$ & Média (DP) & $\mathrm{p}$ \\
& $\mathrm{n}=30$ & $\mathrm{G} 2$ & $\mathrm{p}=30$ \\
\hline Capacidade Funcional & $88,67(11,44)$ & $65,67(23,37)$ & 0,000 \\
Aspecto Físico & $77,50(32,40)$ & $67,50(36,64)$ & 0,273 \\
Dor & $65,80(22,25)$ & $60,13(27,88)$ & 0,469 \\
Estado Geral de Saúde & $81,12(15,12)$ & $66,82(21,50)$ & 0,004 \\
Vitalidade & $75,50(15,67)$ & $63,40(20,88)$ & 0,014 \\
Aspectos Sociais & $88,33(13,90)$ & $68,92(28,32)$ & 0,005 \\
Aspectos Emocionais & $79,99(36,73)$ & $66,65(44,64)$ & 0,221 \\
Saúde Mental & $80,60(16,63)$ & $69,73(20,63)$ & 0,035 \\
GDS & $13,60(1,89)$ & $11,40(2,84)$ & 0,000 \\
\hline
\end{tabular}

$\mathrm{DP}=$ desvio padrão; $\mathrm{n}=$ número de indivíduos. 
Diferença estatisticamente significativa também foi encontrada na comparação dos escores da GDS entre os grupos G1 e G2 $(p=0,000)$. Houve diferença significativa também no número de indivíduos com suspeita de possíveis transtornos depressivos em cada grupo $(\mathrm{p}=0,005)$, $3,3 \%$ no $G 1$ e $33,3 \%$ no $G 2$.

\section{DISCUSSÃO}

A qualidade de vida é um conceito multidimensional que envolve aspectos físicos, sociais e emocionais ${ }^{37}$. Sendo assim, vários estudos têm sido realizados com a finalidade de verificar a QV de diferentes populações em diferentes estágios de suas vidas ${ }^{38}$, tendo grande importância científica e social ${ }^{14}$.

Neste estudo, foi realizada uma comparação da qualidade de vida e depressão entre indivíduos que frequentam grupos de convivência e indivíduos, pareados pela idade, que não frequentam estes grupos.

Os resultados da análise dos aspectos demográficos e clínicos encontrados neste estudo demonstraram que os dois grupos avaliados são estatisticamente semelhantes em relação a estas variáveis. Esta similaridade é importante para permitir a comparação da QV entre os grupos, aumentando a validade interna do estudo.

Os resultados deste estudo demonstraram haver diferença significativa na qualidade de vida entre idosos que participam de grupos de convivência na cidade de Itabira e idosos da mesma idade que não participam destes grupos. Os idosos que participavam de grupos de convivência apresentaram maiores valores nos domínios capacidade funcional, estado geral de saúde, aspectos sociais e saúde mental, avaliados pelo instrumento SF-36, sugerindo que a participação nestes grupos pode ser benéfica para estes aspectos da qualidade de vida.

Um dos principais fatores que podem ter influenciado nesta melhor QV apresentada pelos idosos que participam de grupos de convivência é a socialização. Masuchi \& Kishi ${ }^{39}$ relataram que a socialização é um fator muito importante na determinação da qualidade de vida do idoso. Neri ${ }^{21}$ afirma que a manutenção de relações sociais com amigos da mesma geração favorece o bemestar psicológico e social dos idosos.

Os idosos que participavam de grupos de convivência parecem não apresentar apenas melhor qualidade de vida em relação aos domínios do componente mental. Eles apresentaram também melhor qualidade de vida em relação ao seu componente físico, uma vez que relataram melhor estado geral de saúde e capacidade funcional comparados aos idosos que não participavam de grupos de convivência. Para Rosa et al..$^{40}$, a capacidade funcional pode ser conceituada como grau de preservação do desempenho mantido para desenvolver determinadas atividades do dia a dia ou atitudes. A piora da capacidade funcional está associada a perda de autonomia, dependência e transferência do idoso para uma Instituição de Longa Permanência. Ramos ${ }^{41}$ destaca a importância da avaliação da capacidade funcional como um novo padrão da saúde pública frente ao envelhecimento da população. Sugere-se, assim, que a capacidade funcional constitui importante indicador de bemestar da população estudada, colaborando para uma melhor independência e autonomia. Santos et al. ${ }^{16}$ asseguram que a qualidade de vida do idoso pode estar relacionada à capacidade funcional.

Através do presente estudo, verificaram-se maiores valores nos domínios aspectos físicos, dor e aspectos emocionais do G1, quando comparado ao G2, porém não tendo demonstrado diferença estatisticamente significativa. Assim, parece que outros fatores além da socialização promovida pelo grupo influenciam nestes aspectos $\mathrm{da}$ qualidade de vida. Isto está de acordo com a maior variabilidade encontrada entre os participantes, tanto do grupo G1, quanto do grupo G2, em relação a estes aspectos, quando comparados aos outros aspectos da qualidade de vida.

É possível também que esses aspectos da qualidade de vida não sejam tão responsivos a este tipo de atividade quanto as outras dimensões $d a$ 
qualidade de vida. Em concordância com este resultado, outro estudo mostra que, ao avaliar a qualidade de vida em indivíduos que praticam atividade física, estes três domínios foram os que apresentaram maior variabilidade entre todos os domínios do SF-36 e também não houve diferença estatisticamente significativa entre os grupos em relação a alguns destes domínios. ${ }^{1}$

De acordo com os resultados da pontuação da GDS, houve diferença estatisticamente significativa entre os grupos. No G1, 3,3\% apresentaram suspeita de transtorno depressivo, enquanto esta prevalência no G2 foi de 33,3\%, alcançando seis pontos ou mais. Dessa forma, o estudo sugere que há menor prevalência de depressão nos idosos que frequentam grupos de convivência. Estes dados corroboram com o estudo de Baptista et al..$^{42}$, no qual os resultados apontam que quanto maior o número de atividades sociais praticadas pelos indivíduos, menores os escores de depressão. Vários estudos mostram que a ausência de relacionamentos sociais, qualidade de vida e capacidade funcional podem estar ligados à presença de fatores emocionais, como a depressão.

A socialização promovida pela participação no grupo de convivência pode ser uma explicação para a menor frequência de indivíduos com depressão encontrada neste grupo. Este resultado está de acordo com o estudo de Demura \& Sato ${ }^{43}$, no qual foram avaliados a sintomatologia depressiva, estilo e qualidade de vida em um grupo de 1.302 idosos de ambos os sexos. De acordo com esse estudo, há uma correlação negativa entre a participação em atividades sociais e o número de amigos que os idosos relatavam ter com os sintomas de depressão, isto é, quanto mais amigos e mais atividades sociais, menor a sintomatologia depressiva.

Segundo Chacra ${ }^{44}$, os grupos de convivência estimulam o indivíduo a adquirir maior autonomia, melhora sua autoestima, qualidade de vida, senso de humor e promove sua inclusão social. $\mathrm{O}$ isolamento social pode desencadear consequências no comportamento psicossocial, como medo, ansiedade e depressão. Areosa \&
Ohlweiler ${ }^{45}$ afirmam que a inserção nos grupos de convivência, causa satisfação pessoal, aumento de relacionamentos e o reconhecimento do outro diante do grupo. Em conformidade com este estudo, todos os participantes do G1 afirmam ter $100 \%$ de satisfação com o grupo.

Este foi o primeiro estudo encontrado que comparou a qualidade de vida e depressão entre idosos que participam de grupos de convivência e idosos que não participam destes grupos, controlando as variáveis clínicas e demográficas que podem influenciar nestes desfechos. De acordo com este estudo, idosos que participam de grupos de convivência apresentam melhor QV e menor ocorrência de depressão. Sendo assim, são necessários mais estudos que investiguem a qualidade de vida dos idosos em grupos de convivência com delineamento longitudinal e prospectivo, de forma que possam investigar se existe relação causal entre a participação dos idosos nesses grupos e melhora na qualidade de vida e depressão após o seu ingresso no grupo.

\section{CONCLUSÃO}

No presente estudo pode-se concluir, através dos resultados apontados, que os participantes do Projeto Vida Ativa apresentam melhor qualidade de vida e menor ocorrência de depressão, em comparação a idosos da mesma idade que não participam de grupos de convivência. Desta forma, a criação de grupos de convivência para a terceira idade deve ser difundida e implantada, a fim de contribuir para uma vida com mais autonomia e independência funcional refletindo na melhoria na qualidade de vida e melhor condição emocional durante o envelhecimento.

\section{AGRADECIMENTOS}

Agradecemos aos idosos que aceitaram participar deste estudo, à Secretaria Municipal de Esporte e Lazer que permitiu a nossa presença nos grupos de convivência e a todos que de forma satisfatória participaram na elaboração e conclusão deste estudo. 


\section{REFERÊNCIAS}

1. Acree LS, et al. Physical activity is related to quality of life in older adults. Health and Quality of Life Outcomes 2006, 4:37.

2. Fernandes MGM, et al. O Programa de Atenção à Saúde do Idoso em João Pessoa - PB: realidade e possibilidades. Conc. João Pessoa 2002; 5(7):146-54.

3. Paixão Jr CM, Reichenheim ME. Uma revisão sobre instrumentos de avaliação do estado funcional do idoso. Cad. Saúde Pública 2005, Rio de Janeiro, jan-fev, 21(1):7-19.

4. Seidl EMF, Zannon CMLC. Qualidade de vida e saúde: aspectos conceituais e metodológicos. Cad. Saúde Pública 2004, Rio de Janeiro, marabr, 20(2):580-588

5. Gordilho A, et al. Desafios a serem enfrentados no terceiro milênio pelo setor de saúde na atuação integral ao idoso. Bahia Análise \& Dados 2001; 10(4):138-53.

6. Minayo MCS, Hartz ZMA, Buss PM. Qualidade de vida e saúde: um debate necessário. Ciên Saúde Coletiva 2000; 5(1):7-18.

7. Ministério da Saúde. Programa Nacional de Promoção da Atividade Física "Agita Brasil": Atividade física e sua contribuição para a qualidade de vida. São Paulo. Rev. Saúde Pública 2002, abr ,v.36 n.2.

8. Ciconelli RM, Ferraz MB, Santos W. Tradução para a língua portuguesa e validação do questionário genérico de avaliação de qualidade de vida SF-36 (Brasil SF-36). Rev Bras Reumatol 1998; 39:143-50.

9. Valderrábano F, Jofre R, López-Gómez JM. Quality of life in end-stage renal disease patients. Am J Kidney Dis 2001; 38:443-64.

10. Bulsing FL, et al. A influência dos grupos de convivência sobre a auto-estima das mulheres idosas do município de Santa Cruz do Sul - RS. RBCEH 2007, Passo Fundo, v. 4, n. 1, p. 11-17, jan./jun.

11. França L. Preparação para a aposentadoria: desafios a enfrentar. In: Veras R (org.). Terceira idade: alternativas para uma sociedade em transição. Rio de Janeiro: Relume Dumará/ UNATI; 1999. p. 11-34.

12. Joia LC, Ruiz T, Donalisio MR. Condições associadas ao grau de satisfação com a vida entre a população de idosos. Rev Saúde Pública 2007;41(1):131-8.
13. Organização Mundial de Saúde. Envelhecimento ativo: uma política de saúde. Brasília/ Organização Pan-Americana da Saúde; 2005.

14. Fleck MPA, Chachamovich E, Trentini CM. Projeto WHOQOL-OLD: método e resultados de grupos focais no Brasil. Rev Saúde Pública 2003 37(6):793-9.

15. Bowling A, et al. Let's ask them: a national survey of definitions of quality of life and its enhancement among people aged 65 and over. Int J Aging Hum Dev 2003; 56(4):269-306.

16. Santos SR, et al. Elderly quality of life in the community: application of the Flanagan's Scale. Rev Latino Am Enfermagem 2002; 10(6):757-64.

17. Vecchia RD, Ruiz T, Bocchi SCM, Corrente, JE. Qualidade de vida na terceira idade: um conceito subjetivo. Rev Bras Epidemiol 2005; $8(3): 246-52$.

18. Velarde JE, Avila FC. Methods for quality of life assessment. Salud Pública Méx 2002; 44(4):349-61.

19. Frutuoso D. A terceira idade na universidade. Rio de Janeiro, RJ: Ágora da Ilha, 1999.

20. CNBB. Vida, dignidade e esperança. Fraternidade e pessoas idosas. Campanha da Fraternidade. Texto Base CF 2003. São Paulo: Editora Salesiana; 2002.

21. Neri AL, Sommerhalder C. As várias faces do cuidado e do bem-estar do cuidador. In: Néri AL (Ed.). Cuidar de idosos no contexto da família: questões psicológicas e sociais. Campinas: Alínea. 2001. p. 9-62.

22. Monteiro PP. Envelhecer: histórias, encontros, transformações. Belo Horizonte: Autêntica, 2001.

23. Carneiro RS, et al. Qualidade de vida, apoio social e depressão em idosos: relação com habilidades sociais. Psicologia: Reflexão e Crítica 2007, 20(2):229-237.

24. Capitanini MES. Solidão na velhice: realidade ou mito? In: Néri AL, Freire SA (Orgs.). E por falar em boa velhice. Campinas: Papirus, 2000. p. 69-89.

25. Freire SA. Envelhecimento bem-sucedido e bem-estar psicológico. In: Néri AL, Freire SA (Orgs.). E por falar em boa velhice. Campinas: Papirus, 2000. p. 21-31.

26. Dressler WW, Balieiro MC, Santos JE. The cultural construction of social support in Brazil: associations with health outcomes. Culture, Medicine and Psychiatry 1997, 21, 303- 335. 
27. Gray GR, Ventis DG, Hayslip B. Socio-cognitive skills as a determinant of life satisfaction in aged persons. International Journal of Aging and Human Development 1992, 35(3), 205-218.

28. Nelson ME, et. al. Physical activity and public health in older adults: recommendation from the American College of Sports Medicine and the American Heart Association. Official Journal of the American College of Sports Medicine. 2007.

29. Castro M, et al. Qualidade de vida de pacientes com insuficiência renal crônica em Hemodiálise avaliada através do instrumento genérico SF-36. Rev Assoc Med Bras 2003; 49(3):245-9.

30. Almeida OP, Almeida SA. Confiabilidade da versão brasileira da escala de depressão em geriatria (GDS) versão reduzida. Arq Neuropsiquiatr. 1999;57:421-6.

31. World Health Organization. The ICD-10 Classification of Mental and Behavioural Disorders: diagnostic criteria for research. Geneva: WHO, 1993.

32. American Psychiatric Association. Diagnostic and statistical manual of mental disorders (DSM-IV). 4 Ed. Washington: American Psychiatric Press, 1994.

33. Yesavage JA, et al. Development and validation depression screening scale: a preliminary report. J Psychiatr Res. 1982-1983;17(1):37-49.

34. Almeida OP, Almeida SA. Short versions of the geriatric depression scale: a study of their validity for the diagnosis of a major depressive episode according to ICD-10 and DSM-IV. Int J Geriatr Psychiatry. 1999 Oct;14(10):858-65.

35. Irigaray TQ, Schneider RH. Características de personalidade e depressão em idosas da Universidade para a Terceira Idade (UNITI/ UFRGS). Rev Psiquiatr RS. 2007;29(2).

36. Portney LG, Watkins MP. Reliability. In: Portney LG, Watkins MP. Foundations of clinical research applications to pratice. New Jersey: Prentice-Hall; 2000. p.61-75.

37. Lemos MCD, et al. Qualidade de vida em pacientes com osteoporose: correlação entre OPAQ e SF-36. Rev Bras Reumatol 2006, v. 46, n.5, p. 323-328, set/out.

38. Quadros TMB, et al. Qualidade de vida de acadêmicos do curso de educação física do sexo masculino. Revista Científica JOPEF 2006, 1(4):51-54.

39. Masuchi A, Kishi R. A review of epidemiological studies on the relationship of social networks and support to depressive symptoms in the elderly. Jpn J Public Health 2001, 48:435-448.

40. Rosa TEC, et al. Fatores determinantes da capacidade funcional entre idosos. Rev Saúde Pública 2003, v. 37, n. 1, p. 40-48.

41. Ramos LR. Fatores determinantes do envelhecimento saudável em idosos residentes em centro urbano: Projeto Epidoso, São Paulo, Cad Saúde Pública 2003, Rio de Janeiro, v. 19, n. 13, p. 793-798.

42. Baptista $\mathrm{MN}$, et al. Correlação entre sintomatologia depressiva e prática de atividades sociais em idosos. Avaliação Psicológica, 2006; 5(1):77-85.

43. Demura S, Sato S. Relationships between depression, lifestyle and quality of life in the community dwelling elderly: a comparison between gender and age groups. J Physiol Anthropol Appl Human Sci 2003, 22(3):159-166.

44. Chacra FC. Empatia e comunicação na relação médicopaciente: uma semiologia autopoiética do vínculo. [tese]. Campinas (SP): Faculdade de Ciência Médicas/UNICAMP; 2002.

45. Areosa SVC, Ohlweiler ZNC. Redes, Unisc, v. 5, n. 1, p. 179-187, 2000. 
